\title{
KLEINIAN GROUPS WHICH ARE ALMOST FUCHSIAN
}

\author{
FRANCIS BONAHON
}

\begin{abstract}
We consider the space of all quasifuchsian metrics on the product of a surface with the real line. We show that, in a neighborhood of the submanifold consisting of fuchsian metrics, every non-fuchsian metric is completely determined by the bending data of its convex core.
\end{abstract}

Let $S$ be a surface of finite topological type, obtained by removing finitely many points from a compact surface without boundary, and with negative Euler characteristic. We consider complete hyperbolic metrics on the product $S \times]-\infty, \infty[$.

The simplest ones are the fuchsian metrics defined as follows. Because of our hypothesis that the Euler characteristic of $S$ is negative, $S$ admits a finite area hyperbolic metric, for which $S$ is isometric to the quotient of the hyperbolic plane $\mathbb{H}^{2}$ by a discrete group $\Gamma$ of isometries. The group $\Gamma$ uniquely extends to a group of isometries of the hyperbolic 3 -space $\mathbb{H}^{3}$ respecting the transverse orientation of $\mathbb{H}^{2} \subset \mathbb{H}^{3}$, for which the quotient $\mathbb{H}^{3} / \Gamma$ has a natural identification with $\left.S \times\right]-\infty, \infty[$. A fuchsian metric is any metric on $S \times]-\infty, \infty[$ obtained in this way. Note that the image of $\mathbb{H}^{2}$ in $\mathbb{H}^{3}$ provides in this case a totally geodesic surface in $\left.S \times\right]-\infty, \infty[$, isometric to the original metric on $S$.

These examples can be perturbed to more complex hyperbolic metrics on $S \times]-\infty, \infty[$. See for instance [Th] [Mas]. A quasifuchsian metric on $S \times]-\infty, \infty[$ is one which is obtained by quasi-conformal deformation of a fuchsian metric. Equivalently, a quasifuchsian metric is a geometrically finite hyperbolic metric on $S \times]-\infty, \infty[$ whose cusps exactly correspond to the ends of $S$. These also correspond to the interior points in the space of all hyperbolic metrics on $S \times]-\infty, \infty$ [ for which the ends of $S$ are parabolic [Mar $\operatorname{Su}]$.

If $m$ is a quasifuchsian metric on $S \times]-\infty, \infty$ [, the totally geodesic copy of $S$ which occurred in the fuchsian case is replaced by the convex core $C(m)$, defined as the smallest non-empty closed $m$-convex subset of $S \times]-\infty, \infty[$. If $m$ is not fuchsian, $C(m)$ is 3-dimensional and its boundary consists of two copies of $S$, each facing an end of $S \times]-\infty, \infty[$. The geometry of $\partial C(m)$ was investigated by Thurston [Th1]; see also [EpM]. The component of $\partial C(m)$ that faces the end $S \times\{+\infty\}$ is a pleated surface, totally geodesic almost everywhere, but bent along a family of simple geodesics; this bending is described and quantified by a measured geodesic lamination $\beta^{+}(m)$ on $S$. Similarly, the bending of the negative component of $\partial C(m)$, namely the one facing $S \times\{-\infty\}$, is determined by a measured geodesic lamination $\beta^{-}(m)$.

If $\mathcal{Q}(S)$ denotes the space of isotopy classes of quasifuchsian metrics on $S \times$ ] $-\infty, \infty$ [ and if $\mathcal{M L}(S)$ is the space of measured geodesic laminations on $S$, the

Date: March 25, 2022.

This work was partially supported by grant DMS-0103511 from the National Science Foundation. 
rule $m \mapsto\left(\beta^{+}(m), \beta^{-}(m)\right)$ defines a map $\beta: \mathcal{Q}(S) \rightarrow \mathcal{M L}(S) \times \mathcal{M L}(S)$. By definition, $\beta(m)=(0,0)$ is the metric $m$ is fuchsian, in which case the convex core $C(m)$ is just a totally $m$-geodesic copy of $S$. The image $\beta(m) \in \mathcal{M L}(S)^{2}$, interpreted as a measured geodesic lamination on two copies of $S$, is the bending measured geodesic lamination of the quasifuchsian metric $m$.

The space $\mathcal{Q}(S)$ is a manifold of dimension $2 \theta$ where, if $\chi(S)$ is the Euler characteristic of $S$ and $p$ is its number of ends, $\theta=-3 \chi(S)-p \geqslant 0$. It contains the space $\mathcal{F}(S)$ as a proper submanifold of dimension $\theta$. To some extend, the bending measured lamination $\beta(m)$ measures how far the metric $m \in \mathcal{Q}(S)$ is from being fuchsian. Finally, recall that the space $\mathcal{M L}(S)$ of measured geodesic laminations is a piecewise linear manifold of dimension $\theta$.

Thurston conjectured that the restriction of the bending map to $\beta$ could be used to parametrize $\mathcal{Q}(S)-\mathcal{F}(S)$, namely that it induces a homeomorphism between $\mathcal{Q}(S)-\mathcal{F}(S)$ and an open subset of $\mathcal{M L}(S)^{2}$. The image of $\beta$ was determined in BoO.

The goal of the current paper is to prove Thurston's conjecture on a neighborhood of the space of Fuchsian metrics.

Theorem 1. There exists an open neighborhood $V$ of the fuchsian submanifold $\mathcal{F}(S)$ in $\mathcal{Q}(S)$ such that the bending map $\beta: \mathcal{Q}(S) \rightarrow \mathcal{M L}(S)^{2}$ induces a homeomorphism between $V-\mathcal{F}(S)$ and its image.

There are well-known restrictions for $(\mu, \nu) \in \mathcal{M L}(S)^{2}$ to be in the image of $\beta$; see for instance BoO. In particular, if $(\mu, \nu) \neq(0,0)$ is the bending measured lamination of some $m \in \mathcal{Q}(S)$, then the measured geodesic laminations $\mu$ and $\nu$ must fill up the surface $S$, in the sense that every non-trivial measured geodesic lamination has non-zero geometric intersection number with at least one of $\mu, \nu$. This is equivalent to the condition that every component of $S-\mu \cup \nu$ is, either a topological disk bounded by the union of finitely many geodesic arcs, or a topological annulus bounded on one side by the union of finitely many geodesic arcs and going to a cusp on the other side.

Let $\mathcal{F} \mathcal{M L}(S)$ denote the open subset of $\mathcal{M L}(S)^{2}$ consisting of those $(\mu, \nu)$ where $\mu$ and $\nu$ fill up $S$. Note that $\mathcal{F} \mathcal{M L}(S)$ is endowed with an action of $\mathbb{R}^{+}$, defined by $t(\mu, \nu)=(t \mu, t \nu)$, which decomposes $\mathcal{F} \mathcal{M L}(S)$ as the union of pairwise disjoint rays (=orbits) $] 0, \infty[(\mu, \nu)$.

Theorem 2. In Theorem $\mathbb{Q}$, the neighborhood $V$ of $\mathcal{F}(S)$ and its image $U=\beta(V)$ can be chosen so that $U-\{(0,0)\}$ is an open subset of $\mathcal{F} \mathcal{M L}(S)$ which intersects each ray $] 0, \infty[(\mu, \nu)$ in an interval $] 0, \varepsilon_{\mu \nu}[(\mu, \nu)$.

Theorems 1 and 2 are proved later as Theorem 14. The main idea of the proof is to construct an inverse $\beta^{-1}: U-\{(0,0)\} \rightarrow V-\mathcal{F}(S)$, and splits into two steps: an infinitesimal part, and a transversality argument based on the infinitesimal part. The infinitesimal part is now relatively classical; see for instance Se1]. There are restrictions on which bending data can be realized by an infinitesimal deformation of $m_{0} \in \mathcal{F}(S)$. Through the complex structure of $\mathcal{Q}(S)$, where multiplication by $\mathrm{i}=\sqrt{-1}$ converts bending to shearing, these restrictions can be expressed in a purely 2-dimensional context; see Section 2. The main part of the proof is to show by a transversality argument that any infinitesimal bending data can actually be realized by a deformation. The only significant idea of the paper is to apply the transversality argument, not in $\mathcal{Q}(S)$ where the necessary hypotheses are not 
realized, but in the manifold-with-boundary $\check{\mathcal{Q}}(S)$ obtained by blowing up $\mathcal{Q}(S)$ along the fuchsian submanifold $\mathcal{F}(S)$.

Switching to the blow-up manifold $\mathscr{\mathcal { Q }}(S)$ actually provides a better understanding of the restriction of $\beta$ on a neighborhood of $\mathcal{F}(S)$ and of its inverse. See Theorem 13 for a precise statement.

Acknowledgements: There is strong evidence that the content of this paper has been known to Bill Thurston for about twenty years. I personally learned much of the material of Section 2 many years ago from David Epstein who, I believe, had learned it from Thurston. I am also grateful to Pete Storm and Dick Canary for helping me to clarify my thoughts.

\section{The Earthouake SECTION}

We consider the Teichmüller space $\mathcal{T}(S)$, namely the space of isotopy classes of finite area complete hyperbolic metrics on the surface $S$. Recall that $\mathcal{T}(S)$ is diffeomorphic to $\mathbb{R}^{\theta}$, where $\theta=-3 \chi(S)-p \geqslant 0$ if $\chi(S)$ is the Euler characteristic of $S$ and $p$ is its number of ends.

A standard deformation of a metric $m \in \mathcal{T}(S)$ is the left earthquake $E_{m}^{\mu} \in \mathcal{T}(S)$ along the measured geodesic lamination $\mu$, as constructed in Th2 Ke1 EpM. We consider the infinitesimal left earthquake vector $e_{m}^{\mu}=\frac{d}{d t} E_{m \mid t=0}^{t \mu} \in T_{m} \mathcal{T}(S)$. This provides a section $e^{\mu}: \mathcal{T}(S) \rightarrow T \mathcal{T}(S)$ of the tangent bundle of $\mathcal{T}(S)$, defined by $m \mapsto e_{m}^{\mu}$.

There similarly exists a right earthquake $E_{m}^{-\mu}$ along $\mu$; the notation is justified by the fact that $m \mapsto E_{m}^{-\mu}$ is the inverse of $m \mapsto E_{m}^{\mu}$. We can then consider the infinitesimal right earthquake vector $e_{m}^{-\mu}=\frac{d}{d t} E_{m}^{-t \mu}{ }_{\mid t=0} \in T_{m} \mathcal{T}(S)$ and the corresponding section $e^{-\mu}: \mathcal{T}(S) \rightarrow T \mathcal{T}(S)$ of the tangent bundle of $\mathcal{T}(S)$. Note that $e_{m}^{-\mu}=-e_{m}^{\mu}$, but it will be convenient to keep a separate notation.

Recall that the measured geodesic laminations $\mu, \nu \in \mathcal{M L}(S)$ fill up the surface $S$ if every non-trivial measured geodesic lamination has non-zero geometric intersection number with at least one of $\mu, \nu$. This is equivalent to the condition that every component of $S-\mu \cup \nu$ is, either a topological disk bounded by the union of finitely many geodesic arcs, or a topological annulus bounded on one side by the union of finitely many geodesic arcs and going to a cusp on the other side.

Proposition 3. Let $\mu, \nu \in \mathcal{M L}(S)$ be two non-zero measured geodesic laminations. The intersection of the two sections $e^{\mu}, e^{-\nu}: \mathcal{T}(S) \rightarrow T \mathcal{T}(S)$ of the tangent bundle of $\mathcal{T}(S)$ is transverse. These sections meet in exactly one point if $\mu$ and $\nu$ fill up the surface $S$, and are otherwise disjoint.

Proof. We first translate the problem in terms of the length functions $l_{\mu}, l_{\nu}$ : $\mathcal{T}(S) \rightarrow \mathbb{R}$ which to a metric $m \in \mathcal{T}(S)$ associate the $m$-lengths of the measured geodesic lamination $\mu$ and $\nu$. The Weil-Petersson symplectic form on $\mathcal{T}(S)$ induces an isomorphism between its tangent bundle $T \mathcal{T}(S)$ and its cotangent bundle $T^{*} \mathcal{T}(S)$. A celebrated result of Scott Wolpert Wo1] asserts that this isomorphism sends the section $e^{\mu}$ of $T \mathcal{T}(S)$ to the section $d l_{\mu}$ of $T^{*} \mathcal{T}(S)$. Therefore, Proposition 3 is equivalent to showing that the sections $d l_{\mu}$ and $-d l_{\nu}$ transversely meet in 1 or 0 point, according to whether $\mu$ and $\nu$ fill up the surface $S$ or not.

First consider the case where $\mu$ and $\nu$ fill up $S$. The intersection of the section $d l_{\mu}$ and $-d l_{\nu}$ of $T^{*} \mathcal{T}(S)$ correspond to the points $m \in \mathcal{T}(S)$ where $d_{m} l_{\mu}=-d_{m} l_{\nu}$, namely to the critical points of the function $l_{\mu}+l_{\nu}: \mathcal{T}(S) \rightarrow \mathbb{R}$. It is proved in 
Ke1 Ke2 that, because $\mu$ and $\nu$ fill up $S$, the function $l_{\mu}+l_{\nu}$ admits a unique critical point $m_{0}$. In addition, the hessian of $l_{\mu}+l_{\nu}$ at $m_{0}$ is positive definite Wo2 $\mathrm{Ke} 2$.

Let $u=d_{m_{0}} l_{\mu}=-d_{m_{0}} l_{\nu} \in T_{m_{0}}^{*} \mathcal{T}(S)$. The intersection of the tangent spaces of the sections $d l_{\mu}$ and $-d l_{\nu}$ at $u$ consists of all the vectors of the form $T_{m_{0}}\left(d l_{\mu}\right)(v)=$ $T_{m_{0}}\left(-d l_{\nu}\right)(v)$ for some $v \in T_{m_{0}} \mathcal{T}(S)$, where $T_{m_{0}}\left(d l_{\mu}\right), T_{m_{0}}\left(-d l_{\nu}\right): T_{m_{0}} \mathcal{T}(S) \rightarrow$ $T_{u} T^{*} \mathcal{T}(S)$ denote the tangent maps of the sections $d l_{\mu},-d l_{\nu}: \mathcal{T}(S) \rightarrow T^{*} \mathcal{T}(S)$.

Note that, for an arbitrary $w \in T_{m_{0}} \mathcal{T}(S)$, the vectors $T_{m_{0}}\left(d l_{\mu}\right)(w)$ and $T_{m_{0}}\left(-d l_{\nu}\right)(w)$ both project to $w$ through the tangent map of the projection $T^{*} \mathcal{T}(S) \rightarrow \mathcal{T}(S)$. In particular, the difference $T_{m_{0}}\left(d l_{\mu}\right)(w)-T_{m_{0}}\left(-d l_{\nu}\right)(w)$ is tangent to the fiber, namely is an element of $T_{m_{0}}^{*} \mathcal{T}(S) \subset T_{u} T^{*} \mathcal{T}(S)$. Using local coordinates, one easily sees that this difference is the image of $w$ under the homomorphism $T_{m_{0}} \mathcal{T}(S) \rightarrow T_{m_{0}}^{*} \mathcal{T}(S)$ induced by the hessian of the function $l_{\mu}+l_{\nu}$. (Beware of optimistic simplifications, though: for instance, $T_{m_{0}}\left(-d l_{\nu}\right)$ is not the same as $-T_{m_{0}}\left(d l_{\nu}\right)$ since they take their values in different tangent spaces.) Since the hessian is non-degenerate, this homomorphism is actually an isomorphism.

A consequence of this analysis is that, if $T_{m_{0}}\left(d l_{\mu}\right)(v)=T_{m_{0}}\left(-d l_{\nu}\right)(v)$, then $v$ must be 0 . Therefore, the intersection of the two sections $d l_{\mu}$ and $-d l_{\nu}$ of $T^{*} \mathcal{T}(S)$ at the point $u$ is transverse, since these sections have dimension $\theta$ and the total space $T^{*} \mathcal{T}(S)$ has dimension $2 \theta$. By Weil-Petersson duality, this proves that the sections $e^{\mu}$ of $e^{-\nu}$ of $T \mathcal{T}(S)$ have a transverse intersection consisting of exactly one point.

We now consider the case where $\mu$ and $\nu$ do not fill up the surface. In this case, the function $l_{\mu}+l_{\nu}$ has no critical point [Ke2] (see also the discussion in [Se1, §4.2]). By the above translation, it follows that the sections $e^{\mu}, e^{-\nu}$ are disjoint.

This concludes the proof of Proposition 3 .

If the measured geodesic laminations $\mu, \nu$ fill up the surface $S$, let $\kappa(\mu, \nu)$ denote the (unique) critical point of the length function $l_{\mu}+l_{\nu}: \mathcal{T}(S) \rightarrow \mathbb{R}$. As above, $\kappa(\mu, \nu)$ is also the unique $m \in \mathcal{T}(S)$ such that $e_{m}^{\mu}=e_{m}^{-\nu}$.

Lemma 4. If $\kappa\left(\mu^{\prime}, \nu\right)=\kappa(\mu, \nu)$, then $\mu^{\prime}=\mu$.

Proof. By an (easy) infinitesimal version of [Th2] (see also [Ke2]), an infinitesimal left earthquake completely determines the measured geodesic lamination along which it is performed. If $e_{m}^{\mu^{\prime}}=e_{m}^{-\nu}=e_{m}^{\mu}$, it follows that $\mu^{\prime}=\mu$.

We will need a result similar to Proposition 3 in the unit tangent bundle $T^{1} \mathcal{T}(S)$. Recall that the fibers $T_{m}^{1} \mathcal{T}(S)$ of this bundle are the quotient of $T_{m} \mathcal{T}(S)-\{0\}$ under the equivalence relation which identifies $v$ to $t v$ when $t \in] 0, \infty[$. In particular, $T^{1} \mathcal{T}(S)$ is a manifold of dimension $2 \theta-1$. Let $\bar{e}^{\mu}, \bar{e}^{-\nu}: \mathcal{T}(S) \rightarrow T^{1} \mathcal{T}(S)$ be the sections induced by $e^{\mu}$ and $e^{-\nu}$.

Proposition 5. Let $\mu, \nu \in \mathcal{M L}(S)$ be two non-zero measured geodesic laminations. The intersection of the two sections $\bar{e}^{\mu}, \bar{e}^{-\nu}: \mathcal{T}(S) \rightarrow T^{1} \mathcal{T}(S)$ of the unit tangent bundle of $\mathcal{T}(S)$ is transverse. If $\mu$ and $\nu$ fill up the surface $S$, these sections meet along a section above a line $K(\mu, \nu)$ properly embedded in $\mathcal{T}(S)$. If $\mu, \nu$ do not fill up $S$, the intersection is empty.

Proof. The two sections meet above $m \in \mathcal{T}(S)$ if $\bar{e}_{m}^{\mu}=\bar{e}_{m}^{-\nu}$, namely if there is a $t>0$ such that $e_{m}^{-\nu}=t e_{m}^{\mu}=e_{m}^{t \mu}$. By Proposition 3, this can occur only when $t \mu$ 
and $\nu$ fill the surface, namely only when $\mu$ and $\nu$ fill the surface. Consequently, the two sections have empty intersection if $\mu$ and $\nu$ do not fill up the surface.

If $\mu$ and $\nu$ fill up the surface then, for every $t>0$, Proposition 3 shows that there is a unique $m=\kappa(t \mu, \nu) \in \mathcal{T}(S)$ such that $e_{m}^{t \mu}=e_{m}^{-\nu}$. As a consequence, the two sections $\bar{e}^{\mu}, \bar{e}^{-\nu}: \mathcal{T}(S) \rightarrow T^{1} \mathcal{T}(S)$ meet exactly above the image $K(\mu, \nu)$ of the map $] 0, \infty[\rightarrow \mathcal{T}(S)$ defined by $t \mapsto \kappa(t \mu, \nu)$.

If $m=\kappa(t \mu, \nu)$ so that $e_{m}^{t \mu}=e_{m}^{-\nu}$, the tangent space $T_{e_{m}^{t \mu}} T \mathcal{T}(S)$ is the sum of the tangent spaces $T_{m} e^{t \mu}\left(T_{m} \mathcal{T}(S)\right)$ and $T_{m} e^{-\nu}\left(T_{m} \mathcal{T}(S)\right)$ of the sections $e^{t \mu}$ and $e^{-\nu}$, by the transversality property of Proposition 3 . Therefore, in the unit tangent bundle, the tangent space $T_{\bar{e}_{m}^{\mu}} T^{1} \mathcal{T}(S)$ is the sum of $T_{m} \bar{e}^{\mu}\left(T_{m} \mathcal{T}(S)\right)$ and $T_{m} \bar{e}^{-\nu}\left(T_{m} \mathcal{T}(S)\right)$. As a consequence, the intersection of the two sections $\bar{e}^{\mu}$ and $\bar{e}^{-\nu}$ is transverse above the point $m=\kappa(t \mu, \nu)$.

By transversality, the intersection of the sections is a submanifold of the image of $\bar{e}^{\mu}$. Its dimension is equal to 1 , by consideration of the dimensions of $\mathcal{T}(S)$ and $T^{1} \mathcal{T}(S)$. Since the projection $\bar{e}^{\mu}(\mathcal{T}(S)) \rightarrow \mathcal{T}(S)$ is a diffeomorphism, it follows that the projection $K(\mu, \nu)$ of this projection is a 1-dimensional submanifold of $\mathcal{T}(S)$.

By definition, $\kappa(t \mu, \nu)$ is the unique minimum of the convex function $t d l_{\mu}+d l_{\nu}$, which has positive hessian at this minimum. It follows that $\kappa(t \mu, \nu)$ is a continuous function of $t$. Conversely, $t$ is completely determined by $m=\kappa(t \mu, \nu)$ by Lemma 4 . As a consequence, if $m=\kappa(t \mu, \nu)$ stays in a bounded subset of $\mathcal{T}(S)$, then $t$ stays in a compact subset of $] 0, \infty[$. In other words, the map ]0, $\infty[\rightarrow \mathcal{T}(S)$ defined by $t \mapsto \kappa(t \mu, \nu)$ is injective, continuous and proper. It follows that its image $K(\mu, \nu)$, which we already know is a 1 -dimensional submanifold of $\mathcal{T}(S)$, is a line properly embedded in $\mathcal{T}(S)$.

Following the terminology of [Se1] (motivated by [Ke2]), let the Kerckhoff line be the proper 1-dimensional submanifold $K(\mu, \nu) \subset \mathcal{T}(S)$, consisting of all the $\kappa(t \mu, \nu)$ with $t>0$.

\section{NeCESSary CONDITION FOR SMALl BENDing}

Let $t \mapsto m_{t}, t \in\left[0, \varepsilon\left[\right.\right.$, be a small differentiable curve in $\mathcal{Q}(S)$. If $\beta\left(m_{t}\right)=$ $\left(\beta^{+}(t), \beta^{-}(t)\right) \in \mathcal{M L}(S)^{2}$ is its bending measured geodesic lamination, it is shown in Bo3] that the right derivative $\frac{d}{d t^{+}} \beta\left(m_{t}\right)_{\mid t=0}$ exists, as an element of the tangent space of $\mathcal{M L}(S)^{2}$ at $\beta\left(m_{0}\right)$. In general, because $\mathcal{M L}(S)$ is not a differentiable manifold, this tangent space consists of geodesic laminations with a transverse structure which is less regular than a transverse measure Bo2]. However, if we assume in addition that the starting point $m_{0}$ of the curve is fuchsian, the tangent space of $\mathcal{M L}(S)^{2}$ at $\beta\left(m_{0}\right)=(0,0)$ is just $\mathcal{M L}(S)^{2}$; see [Bo2].

We can therefore consider the converse problem: Given a fuchsian metric $m_{0} \in$ $\mathcal{F}(S)$ and a pair $(\mu, \nu) \in \mathcal{M L}(S)^{2}$ of measured geodesic laminations, does there exist a small differentiable curve $t \mapsto m_{t} \in \mathcal{Q}(S), t \in\left[0, \varepsilon\left[\right.\right.$, originating from $m_{0}$ and such that $\frac{d}{d t^{+}} \beta\left(m_{t}\right)_{\mid t=0}=(\mu, \nu)$ ? The following result shows that $m_{0}$ is completely determined by $\mu$ and $\nu$.

Note that, by construction, there is a natural identification between the submanifold $\mathcal{F}(S) \subset \mathcal{Q}(S)$ consisting of all fuchsian metrics and the Teichmüller space $\mathcal{T}(S)$. 
Proposition 6. Let $\mu, \nu \in \mathcal{M L}(S)$ be two measured geodesic laminations, and let $t \mapsto m_{t}, t \in[0, \varepsilon[$ be a differentiable curve in $\mathcal{Q}(S)$, originating from a fuchsian metric $m_{0}$ and such that the derivative $\frac{d}{d t^{+}} \beta\left(m_{t}\right)_{\mid t=0}$ of the bending measured lamination is equal to $(\mu, \nu)$. Then $\mu$ and $\nu$ fill up the surface $S$, and $m_{0} \in \mathcal{F}(S)=\mathcal{T}(S)$ is equal to the minimum $\kappa(\mu, \nu)$ of the length function $l_{\mu}+l_{\nu}: \mathcal{T}(S) \rightarrow \mathbb{R}$.

Proof. We consider two other curves in $\mathcal{Q}(S)$.

The first one is the pure bending $t \mapsto B_{m_{0}}^{t \mu}$, obtained by bending the surface $S$ along the measured geodesic lamination $t \mu$ while keeping the metric induced on this pleated surface equal to $m_{0}$. For $t \geqslant 0$ small enough, $B_{m_{0}}^{t \mu}$ is a quasifuchsian metric for which the positive side of the boundary $\partial C\left(B_{m_{0}}^{t \mu}\right)$ is a pleated surface with induced metric $m_{0}$ and with bending measured geodesic lamination $t \mu$. See EpM, §3] or [Bo1] for the construction of $B_{m_{0}}^{t \mu}$, and [Mar, §9] to guarantee that it is quasifuchsian for $t$ sufficiently small. In addition, it is proved in [EpM, §3.9] Bo1 that this curve is differentiable in $\mathcal{Q}(S)$, and in particular admits a tangent vector $b_{m_{0}}^{\mu}=\frac{d}{d t} B_{m_{0} \mid t=0}^{t \mu} \in T_{m_{0}} \mathcal{Q}(S)$. This tangent vector $b_{m_{0}}^{\mu}$ is the infinitesimal pure bending of $m_{0} \in \mathcal{F}(S)$ along the measured geodesic lamination $\mu$.

The second curve will use the shear-bend coordinates associated, as in [Bo1], to a maximal geodesic lamination $\lambda$ containing the support of $\mu$. These coordinates provide a local parametrization of $\mathcal{Q}(S)$ in terms of the geometry of a pleated surface with pleating locus $\lambda$. Let $m_{t}^{\prime}$ correspond to a pleated surface whose induced metric is equal to the metric $m_{t}^{+} \in \mathcal{T}(S)$ induced on the positive component of the boundary $\partial C\left(m_{t}\right)$ of the convex core, and whose bending data is equal to $t \mu$. By Mar, §9], $m_{t}$ is a quasifuchsian metric for $t$ small.

It is proved in Bo3] that, because the curve $t \mapsto m_{t}$ is differentiable and because $\frac{d}{d t^{+}} \beta^{+}\left(m_{t}\right)_{\mid t=0}=\mu$, the right derivative $\dot{m}_{0}^{+}=\frac{d}{d t} m_{t \mid t=0}^{+} \in T_{m_{0}} \mathcal{T}(S)$ exists and the two curves $t \mapsto m_{t}$ and $t \mapsto m_{t}^{\prime}$ have the same tangent vector at $t=0$. In particular, by differentiability of the shear-bend coordinates, the tangent vector $\dot{m}_{0}=\frac{d}{d t} m_{t \mid t=0}=\frac{d}{d t} m_{t \mid t=0}^{\prime} \in T_{m_{0}} \mathcal{Q}(S)$ is the sum of $b_{m_{0}}^{\mu}$ and of $\dot{m}_{0}^{+}=\frac{d}{d t} m_{t \mid t=0}^{+} \in$ $T_{m_{0}} \mathcal{T}(S)=T_{m_{0}} \mathcal{F}(S)$.

Similarly, bending $S$ in the negative direction, we can define the infinitesimal pure bending vector $b_{m_{0}}^{-\nu}=-b_{m_{0}}^{\nu} \in T_{m_{0}} \mathcal{Q}(S)$ of $m_{0}$ along $-\nu$. We can also consider the metric $m_{t}^{-} \in \mathcal{T}(S)$ induced on the negative side of $\partial C\left(m_{t}\right)$. Then, as above, the vector $\dot{m}_{0}$ is the sum of $b_{m_{0}}^{-\nu}$ and of $\dot{m}_{0}^{-}=\frac{d}{d t} m_{t \mid t=0}^{-} \in T_{m_{0}} \mathcal{T}(S)=T_{m_{0}} \mathcal{F}(S)$.

Finally, we will use the complex structure of $\mathcal{Q}(S)$ coming from the fact that the isometry group of $\mathbb{H}^{3}$ is $\mathrm{PSL}_{2}(\mathbb{C})$. Indeed, considering the holonomy of hyperbolic metrics embeds $\mathcal{Q}(S)$ into the space $\mathcal{R}(S)$ of (conjugacy classes) of representations $\pi_{1}(S) \rightarrow \mathrm{PSL}_{2}(\mathbb{C})$. This representation space $\mathcal{R}(S)$ is a complex manifold near the image of $\mathcal{Q}(S)$, and admits this image as a complex submanifold (an open subset if $S$ is compact). The shear-bend local coordinates are well behaved with respect to this complex structure; see Bo1. In particular, multiplication by $\mathrm{i}=\sqrt{-1}$ exchanges shearing and bending. We will use the following two consequences of this. First of all, at a fuchsian metric $m_{0}$, the tangent space $T_{m_{0}} \mathcal{Q}(S)$ is the direct sum of $T_{m_{0}} \mathcal{F}(S)$ and of $\mathrm{i} T_{m_{0}} \mathcal{F}(S)$. In addition, the infinitesimal pure bending vector $b_{m_{0}}^{\mu}$ belongs to i $T_{m_{0}} \mathcal{F}(S)$ and is equal to $e_{m_{0}}^{\mu}$, where $e_{m_{0}}^{\mu} \in T_{m_{0}} \mathcal{T}(S)=T_{m_{0}} \mathcal{F}(S)$ is the infinitesimal earthquake vector along $\mu$.

Applying the decomposition $T_{m_{0}} \mathcal{Q}(S)=T_{m_{0}} \mathcal{F}(S) \oplus \mathrm{i} T_{m_{0}} \mathcal{F}(S)$ to the vector $\dot{m}_{0}=\dot{m}_{0}^{+}+b_{m_{0}}^{\mu}=\dot{m}_{0}^{-}+b_{m_{0}}^{-\nu}$, we conclude that $b_{m_{0}}^{\mu}=b_{m_{0}}^{-\nu}$. Multiplying by $-\mathrm{i}$, it 
follows that $e_{m_{0}}^{\mu}=e_{m_{0}}^{-\nu}$. As in the proof of Proposition 3, this is equivalent to the property that $m_{0}=\kappa(\mu, \nu)$.

\section{REALIZING SMALL BENDiNG}

The goal of this section is to prove a converse to Proposition 6 , by constructing in Proposition 11 a small curve of quasifuchsian metrics $t \mapsto m_{t} \in \mathcal{Q}(S), t \in[0, \varepsilon[$, such that $\beta\left(m_{t}\right)=(t \mu, t \nu)$ for every $t$.

For the measured geodesic lamination $\mu \in \mathcal{M L}(S)$, let $\mathcal{P}^{+}(\mu)$ (resp. $\mathcal{P}^{-}(\mu)$ ) be the space of quasifuchsian metrics $m$ such that the positive (resp. negative) component of the convex core boundary $\partial C(\mathrm{~m})$ has bending measured geodesic lamination $t \mu$, for some $t \in[0, \infty[$.

Recall that $\theta=-3 \chi(S)+p$ denotes the dimension of the Teichmüller space $\mathcal{T}(S)$.

Lemma 7. The space $\mathcal{P}^{ \pm}(\mu)$ is a submanifold-with-boundary of $\mathcal{Q}(S)$, with dimension $\theta+1$ and with boundary $\mathcal{F}(S)$.

Proof. We can use the coordinates developed in Bo1, and associated to a maximal geodesic lamination $\lambda$ containing the support of $\mu$. These provide an open differentiable embedding $\varphi: \mathcal{Q}(S) \rightarrow \mathcal{T}(S) \times \mathcal{H}_{0}(\lambda ; \mathbb{R} / 2 \pi \mathbb{Z})$. The first component of $\varphi(m)$ is the hyperbolic metric induced on the unique $m$-pleated surface $f_{m}$ with pleating locus $\lambda$. The second component is the bending transverse cocycle of $f_{m}$, which belongs to the topological group $\mathcal{H}_{0}(\lambda ; \mathbb{R} / 2 \pi \mathbb{Z}) \cong(\mathbb{R} / 2 \pi \mathbb{Z})^{\theta} \oplus \mathbb{Z} / 2$ of all $(\mathbb{R} / 2 \pi \mathbb{Z})$-valued transverse cocycles for $\lambda$ that satisfy a certain cusp condition. In general, the bending of the pleated surface $f_{m}$ is measured by a transverse cocycle and not by a measured geodesic lamination because $f_{m}$ is not necessarily locally convex.

For notational convenience, it is useful to lift $\varphi$ to an embedding $\psi: \mathcal{Q}(S) \rightarrow$ $\mathcal{T}(S) \times \mathcal{H}_{0}(\lambda ; \mathbb{R})$, such that $\psi$ sends $\mathcal{F}(S) \cong \mathcal{T}(S)$ to $\mathcal{T}(S) \times\{0\}$ by the identity. Here, $\mathcal{H}_{0}(\lambda ; \mathbb{R}) \cong \mathbb{R}^{\theta}$ denotes the space of $\mathbb{R}$-valued transverse cocycle satisfying the cusp condition. Such a $\psi$ exists and is unique because $\mathcal{Q}(S)$ is simply connected.

The vector space $\mathcal{H}_{0}(\lambda ; \mathbb{R})$ contains the transverse measure (also denoted by $\mu$ ) of the measured geodesic lamination $\mu$, and therefore also contains the two rays $[0, \infty[\mu$ and $]-\infty, 0] \mu$, consisting of all positive (resp. negative) real multiples of $\mu$.

We claim that $\mathcal{P}^{+}(\mu)$ locally corresponds, under $\psi$, to the intersection of $\psi(\mathcal{Q}(S))$ with $\mathcal{T}(S) \times\left[0, \infty\left[\mu\right.\right.$. Clearly, $\psi$ sends an element of $\mathcal{P}^{+}(\mu)$ to $\mathcal{T}(S) \times$ $[0, \infty[\mu$. Conversely, it is proved in KaT] that the map $\eta: \mathcal{Q}(S) \rightarrow \mathcal{T}(S) \times \mathcal{M L}(S)$ which, to a quasifuchsian metric $m \in \mathcal{Q}(S)$, associates the induced metric $m^{+} \in$ $\mathcal{T}(S)$ and the bending measured lamination $\beta^{+}(m)$ of the positive boundary component of the convex core $C(m)$ is a local homeomorphism. In addition, interpreting the ray $\left[0, \infty\left[\mu\right.\right.$ as a subset of both $\mathcal{H}_{0}(\lambda ; \mathbb{R})$ and $\mathcal{M L}(S)$, the local inverse for $\eta$ constructed in KaT] coincides with $\psi$ on $\mathcal{T}(S) \times[0, \infty[\mu$. It follows that, if $m \in \mathcal{Q}(S)$ is sufficiently close to $m_{0} \in \mathcal{P}^{+}(\mu)$ and if $\psi(m) \in \mathcal{T}(S) \times[0, \infty[\mu$, the bending measured lamination $\beta^{+}(\mu)$ is equal to the second component $t \mu$ of $\psi(m)$. Therefore, a metric $m$ near $m_{0} \in \mathcal{P}^{+}(\mu)$ is in $\mathcal{P}^{+}(\mu)$ if and only if $\psi(m) \in \mathcal{T}(S) \times[0, \infty[\mu$.

A similar property holds for $\mathcal{P}^{-}(\mu)$ by symmetry. Therefore, under the diffeomorphism $\psi, \mathcal{P}^{+}(\mu), \mathcal{P}^{-}(\mu)$ and $\mathcal{F}(S)$ locally correspond to the intersection of $\psi(\mathcal{Q}(S))$ with $\mathcal{T}(S) \times[0, \infty[\mu, \mathcal{T}(S) \times]-\infty, 0] \mu$ and $\mathcal{T}(S) \times\{0\}$, respectively. 
Given two measured geodesic laminations $\mu, \nu \in \mathcal{M L}(S)$, we want to consider the intersection of $\mathcal{P}^{+}(\mu)$ and $\mathcal{P}^{-}(\nu)$. Note that this intersection is far from being transverse, since these two $(\theta+1)$-dimensional submanifolds both contain the $\theta$-dimensional submanifold $\mathcal{F}(S)$ as their boundary. For this reason, we will consider the manifold-with-boundary $\check{\mathcal{Q}}(S)$ obtained by blowing-up $\mathcal{Q}(S)$ along the submanifold $\mathcal{F}(S)$. Namely, $\mathscr{\mathcal { Q }}(S)$ is the union of $\mathcal{Q}(S)-\mathcal{F}(S)$ and of the unit normal bundle $N^{1} \mathcal{F}(S)$ with the appropriate topology. Recall that the normal bundle $N \mathcal{F}(S) \rightarrow \mathcal{F}(S)$ is intrinsically defined as the bundle whose fiber $N_{m} \mathcal{F}(S)$ at $m \in \mathcal{F}(S)$ is the quotient $T_{m} \mathcal{Q}(S) / T_{m} \mathcal{F}(S)$, and that the fiber $N_{m}^{1} \mathcal{F}(S)$ of the unit normal bundle $N^{1} \mathcal{F}(S) \rightarrow \mathcal{F}(S)$ is the quotient of $N_{m} \mathcal{F}(S)-\{0\}$ under the multiplicative action of $\mathbb{R}^{+}$. Considering a tubular neighborhood of $\mathcal{F}(S), \check{\mathcal{Q}}(S)$ is easily endowed with a natural structure of differentiable manifold with boundary $N^{1} \mathcal{F}(S)$.

Exploiting the complex structure of $\mathcal{Q}(S)$, identify the normal bundle $N \mathcal{F}(S)$ to $\mathrm{i} T \mathcal{F}(S)$. The inclusion map $\mathcal{P}^{+}(\mu)-\mathcal{F}(S) \rightarrow \mathcal{Q}(S)-\mathcal{F}(S)$ uniquely extends to an embedding $\mathcal{P}^{+}(\mu) \rightarrow \check{\mathcal{Q}}(S)$, which to $m \in \mathcal{F}(S)=\partial \mathcal{P}^{+}(\mu)$ associates the unit normal vector $\bar{b}_{m}^{\mu} \in N^{1} \mathcal{F}(S)$ which is in the direction of the infinitesimal pure bending vector $b_{m}^{\mu}$ of $m$ along $+\mu$. We can similarly define an embedding $\mathcal{P}^{-}(\nu) \rightarrow \check{\mathcal{Q}}(S)$, by associating to $m \in \mathcal{F}(S)$ the unit normal vector $-\bar{b}_{m}^{\nu} \in N^{1} \mathcal{F}(S)$ which is in the direction of the infinitesimal pure bending vector $b_{m}^{-\nu}=-b_{m}^{\nu}$ of $m$ along $-\nu$. Let $\check{\mathcal{P}}^{+}(\mu)$ and $\check{\mathcal{P}}^{-}(\nu) \subset \check{\mathcal{Q}}(S)$ be the respective images of these embeddings.

Lemma 8. The subspaces $\check{\mathcal{P}}^{+}(\mu)$ and $\check{\mathcal{P}}^{-}(\nu)$ are submanifolds of $\check{\mathcal{Q}}(S)$, with boundary contained in $\partial \check{\mathcal{Q}}(S)$.

Proof. The embedding $\psi: \mathcal{Q}(S) \rightarrow \mathcal{T}(S) \times \mathcal{H}_{0}(\lambda ; \mathbb{R})$ of the proof of Lemma 7 sends the vector $b_{m}^{\mu}$ to the vector $(0, \mu)$ in the tangent space of $\mathcal{T}(S) \times \mathcal{H}_{0}(\lambda ; \mathbb{R})$. The result then immediately follows from the fact that $\psi$ locally identifies $\mathcal{P}^{+}(\mu), \mathcal{P}^{-}(\mu)$ and $\mathcal{T}(S)$ to $\mathcal{T}(S) \times[0, \infty[\mu, \mathcal{T}(S) \times]-\infty, 0] \mu$ and $\mathcal{T}(S) \times\{0\}$, respectively. This proves Lemma 0 .

Proposition 9. The boundaries $\partial \check{\mathcal{P}}^{+}(\mu)$ and $\partial \check{\mathcal{P}}^{-}(\nu)$ have a non-empty intersection if and only if $\mu$ and $\nu$ fill up the surface $S$. If $\mu$ and $\nu$ fill up $S$, the intersection of $\partial \check{\mathcal{P}}^{+}(\mu)$ and $\partial \check{\mathcal{P}}^{-}(\nu)$ in $\partial \mathscr{\mathcal { Q }}(S)$ is transverse, and is equal to the image of the Kerckhoff line $K(\mu, \nu)$ under the section $m \mapsto \bar{b}_{m}^{\mu}=-\bar{b}_{m}^{\nu} \in \partial \check{\mathcal{Q}}(S)$.

Proof. The diffeomorphism $T^{1} \mathcal{T}(S)=T^{1} \mathcal{F}(S) \rightarrow \mathrm{i} T^{1} \mathcal{F}(S)=N^{1} \mathcal{F}(S)=\partial \check{\mathcal{Q}}(S)$ defined by multiplication by i sends $\bar{e}_{m}^{\mu}$ to $\bar{b}_{m}^{\mu}$ and $\bar{e}_{m}^{-\nu}$ to $\bar{b}_{m}^{-\nu}$. This translates Proposition 9 to a simple rephrasing of Proposition 5 .

An immediate consequence of Proposition 9 is that the intersection of the two $(\theta+1)$-dimensional submanifolds $\check{\mathcal{P}}^{+}(\mu)$ and $\check{\mathcal{P}}^{-}(\nu)$ in $\check{\mathcal{Q}}(S)$ is transverse near the boundary $\partial \check{\mathcal{Q}}(S)$. In particular, the intersection $\check{\mathcal{P}}^{+}(\mu) \cap \check{\mathcal{P}}^{-}(\nu)$ is a 2 -dimensional submanifold of $\check{\mathcal{Q}}(S)$ near $\partial \check{\mathcal{Q}}(S)$, with boundary $\partial \check{\mathcal{P}}^{+}(\mu) \cap \partial \check{\mathcal{P}}^{-}(\nu)$ contained in $\partial \mathcal{Q}(S)$.

By definition, a metric $m \in \mathcal{P}^{+}(\mu) \cap \mathcal{P}^{-}(\nu)$ has bending measured lamination $\beta(m)=(t \mu, u \nu)$ for some $t, u \geqslant 0$. This gives a differentiable map $\pi: \mathcal{P}^{+}(\mu) \cap$ $\mathcal{P}^{-}(\nu) \rightarrow \mathbb{R}^{2}$, defined by $m \mapsto(t, u)$.

Let $\check{\mathbb{R}}^{2}$ be obtained by blowing up $\mathbb{R}^{2}$ along $\{0\}$. Because $\pi^{-1}(0)=\mathcal{F}(S)$, the map $\pi$ lifts to a differentiable map $\check{\pi}: \check{\mathcal{P}}^{+}(\mu) \cap \check{\mathcal{P}}^{-}(\nu) \rightarrow \check{\mathbb{R}}^{2}$. 
Lemma 10. The map $\check{\pi}: \check{\mathcal{P}}^{+}(\mu) \cap \check{\mathcal{P}}^{-}(\nu) \rightarrow \check{\mathbb{R}}^{2}$ is a local diffeomorphism near $\partial \check{\mathcal{P}}^{+}(\mu) \cap \partial \check{\mathcal{P}}^{-}(\nu)$

Proof. We will prove that, at any point $p_{0} \in \partial \check{\mathcal{P}}^{+}(\mu) \cap \partial \check{\mathcal{P}}^{-}(\nu)$, the (linear) tangent map $T_{p_{0}} \check{\pi}: T_{p_{0}} \check{\mathcal{P}}^{+}(\mu) \cap T_{p_{0}} \check{\mathcal{P}}^{-}(\nu) \rightarrow T_{\check{\pi}\left(p_{0}\right)} \check{\mathbb{R}}^{2}$ is injective.

Let $v \in T_{p_{0}} \check{\mathcal{P}}^{+}(\mu) \cap T_{p_{0}} \check{\mathcal{P}}^{-}(\nu)$ be such that $T_{p_{0}} \check{\pi}(v)=0$. Considering the map $\mathcal{P}^{+}(\mu) \rightarrow\left[0, \infty\left[\right.\right.$ which to $m \in \mathcal{P}^{+}(\mu)$ associates $\beta^{+}(m) / \mu \in[0, \infty[$ and the induced map $\tilde{\mathcal{P}}^{+}(\mu) \rightarrow[0, \infty[$, we see that $v$ must necessarily be in the tangent space of the boundary $\partial \check{\mathcal{P}}^{+}(\mu)$. Symmetrically, it must be in $T_{p_{0}} \partial \check{\mathcal{P}}^{-}(\nu)$. Therefore, $v$ is tangent to the intersection $\partial \check{\mathcal{P}}^{+}(\mu) \cap \partial \check{\mathcal{P}}^{-}(\nu)$.

Let us analyze the restriction of $\check{\pi}$ to the boundary $\partial \check{\mathcal{P}}^{+}(\mu) \cap \partial \check{\mathcal{P}}^{-}(\nu)$. By Proposition 9, $\partial \check{\mathcal{P}}^{+}(\mu) \cap \partial \check{\mathcal{P}}^{-}(\nu)$ is equal to the image of the Kerckhoff line $K(\mu, \nu)$ under the section $m \mapsto \bar{b}_{m}^{\mu}=-\bar{b}_{m}^{\nu} \in \partial \check{\mathcal{Q}}(S)$. Recall that an element of the Kerckhoff line $K(\mu, \nu)$ is of the form $m=\kappa(t \mu, \nu)$ for some $t>0$, which is equivalent to the property that $b_{m}^{t \mu}=-b_{m}^{\nu}$. We will also need a coordinate chart for $\check{\mathbb{R}}^{2}$ near $\check{\pi}\left(p_{0}\right)$. Noting that the image of $\pi$ is contained in the quadrant $\left[0, \infty\left[^{2}\right.\right.$, we can use for this the chart $\varphi:] 0, \infty\left[\times\left[0, \infty\left[\rightarrow \check{\mathbb{R}}^{2}\right.\right.\right.$ defined on the interior by $(x, y) \mapsto(x y, y)$.

We claim that, if $m=\kappa(t \mu, \nu) \in K(\mu, \nu)$, then $\varphi^{-1} \circ \check{\pi}\left(\bar{b}_{m}^{\mu}\right)$ is just equal to $(t, 0)$. To see this, choose, in the 2 -dimensional manifold $\check{\mathcal{P}}^{+}(\mu) \cap \check{\mathcal{P}}^{-}(\nu)$, a small curve $s \mapsto \check{m}_{s}, s \in\left[0, \varepsilon\left[\right.\right.$, such that $\check{m}_{0}=\bar{b}_{m}^{\mu} \in \partial \check{\mathcal{P}}^{+}(\mu) \cap \partial \check{\mathcal{P}}^{-}(\nu)$ and such that $\frac{d}{d s^{+}} \check{m}_{s \mid t=0}$ is not tangent to the boundary. The curve $s \mapsto \check{m}_{s}$ projects to a differentiable curve $t \mapsto m_{t} \in \mathcal{Q}(S)$ with $m_{0}=m$. By definition of $\mathcal{P}^{+}(\mu)$ and $\mathcal{P}^{-}(\nu)$, the bending measured lamination $\beta\left(m_{s}\right)$ is of the form $(t(s) \mu, u(s) \nu)$ for two differentiable functions $t(s)$, and $u(s)$ with $t(0)=u(0)=0$. Since $\frac{d}{d s^{+}} \check{m}_{s \mid t=0}$ points away from the boundary, the curve $s \mapsto m_{s}$ is not tangent to $\mathcal{F}(S)$ at $s=0$, and it follows that at least one of the derivatives $t^{\prime}(0), u^{\prime}(0)$ is non-trivial. If we apply Proposition 6, we conclude that $m=\kappa\left(t^{\prime}(0) \mu, u^{\prime}(0) \nu\right)$. Since $m=\kappa(t \mu, \nu)$, it follows that $t^{\prime}(0) / u^{\prime}(0)=t$ by Lemma 1 . In particular, $t(s) / u(s)$ tends to $t$ as $s$ tends to 0 . Therefore, $\varphi^{-1} \circ \check{\pi}\left(\bar{b}_{m}^{\mu}\right)$, which is the limit of $\varphi^{-1} \circ \check{\pi}\left(\check{m}_{s}\right)=$ $\varphi^{-1} \circ \pi\left(m_{s}\right)=\varphi^{-1} \circ(t(s), u(s))=(t(s) / u(s), u(s))$ as $s>0$ tends to 0 , is equal to $(t, 0)$.

This computation shows that the restriction of $\check{\pi}$ to the boundary $\partial \check{\mathcal{P}}^{+}(\mu) \cap$ $\partial \check{\mathcal{P}}^{-}(\nu)$ is a diffeomorphism onto its image. In particular, if $v \in T_{p_{0}} \partial \check{\mathcal{P}}^{+}(\mu) \cap$ $T_{p_{0}} \partial \mathcal{P}^{-}(\nu)$ is such that $T_{p_{0}} \check{\pi}(v)=0$, then necessarily $v=0$.

This concludes the proof that the tangent map $T_{p_{0}} \check{\pi}: T_{p_{0}} \check{\mathcal{P}}^{+}(\mu) \cap T_{p_{0}} \check{\mathcal{P}}^{-}(\nu) \rightarrow$ $T_{\check{\pi}\left(p_{0}\right)} \check{\mathbb{R}}^{2}$ is injective. Since $\check{\pi}$ sends the boundary of the 2 -dimensional manifold $\check{\mathcal{P}}^{+}(\mu) \cap \check{\mathcal{P}}^{-}(\nu)$ to the boundary of the 2 -dimensional manifold $\check{\mathbb{R}}^{2}$, this proves that $\check{\pi}: \check{\mathcal{P}}^{+}(\mu) \cap \check{\mathcal{P}}^{-}(\nu) \rightarrow \check{\mathbb{R}}^{2}$ is a local diffeomorphism near $p_{0} \in \partial \check{\mathcal{P}}^{+}(\mu) \cap \partial \check{\mathcal{P}}^{-}(\nu)$.

This immediately gives the following converse to Proposition 6 .

Proposition 11. Let $\mu, \nu \in \mathcal{M L}(S)$ be two measured geodesic laminations which fill up the surface $S$, and let $m_{0}$ be the minimum $\kappa(\mu, \nu)$ of the length function $l_{\mu}+l_{\nu}$. Then there is a small differentiable curve $t \mapsto m_{t} \in \mathcal{Q}(S), t \in[0, \varepsilon[$, beginning at $m_{0}$ and such that the bending measured lamination $\beta\left(m_{t}\right)$ is equal to $(t \mu, t \nu)$ for every $t$.

Proof. Consider the curve $t \mapsto(t, t), t \in\left[0, \varepsilon\left[\right.\right.$, in $\mathbb{R}^{2}$. By Lemma 10, for $\varepsilon$ small enough, there is a curve $t \mapsto \check{m}_{t} \in \check{\mathcal{P}}^{+}(\mu) \cap \check{\mathcal{P}}^{-}(\nu)$ such that $t \mapsto \check{\pi}\left(\check{m}_{t}\right)$ coincides 
with the lift of $t \mapsto(t, t)$ to $\check{\mathbb{R}}^{2}$. By definition of the map $\pi$, this just means that the projection $m_{t} \in \mathcal{Q}(S)$ of $\check{m}_{t} \in \check{\mathcal{Q}}(S)$ is such that $\beta\left(m_{t}\right)=(t \mu, t \nu)$.

\section{Parametrizing QUASi-Fuchsian Groups By THEIR SMAll Bending}

Recall that $\mathcal{F} \mathcal{M L}(S)$ denotes the open subset of $\mathcal{M L}(S)^{2}$ consisting of those pairs $(\mu, \nu)$ such that $\mu$ and $\nu$ fill up the surface $S$.

Let $\check{\mathcal{F}} \mathcal{M L}(S)$ be obtained by blowing up $\mathcal{F} \mathcal{M L}(S) \cup\{(0,0)\}$ along $\{(0,0)\}$. Namely, $\check{\mathcal{F}} \mathcal{M L}(S)$ is formally obtained from $\mathcal{F} \mathcal{M L}(S)$ by extending each ray ] $0, \infty[(\mu, \nu)$ to a semi-open ray $[0, \infty[(\mu, \nu)$, with the obvious topology. Note that the boundary $\partial \check{\mathcal{F}} \mathcal{M L}(S)$ is just the quotient space of $\mathcal{F} \mathcal{M L}(S)$ under the multiplicative action of $\mathbb{R}^{+}$.

For every $(\mu, \nu) \in \mathcal{F} \mathcal{M L}(S)$, Proposition 11 provides a maximal ray $R_{\mu \nu}=$ $\left[0, \varepsilon_{\mu \nu}\left[(\mu, \nu)\right.\right.$ in $\mathcal{F} \mathcal{M L}(S) \cup\{(0,0)\}$ and a differentiable map $\Phi_{\mu \nu}: R_{\mu \nu} \rightarrow Q(S)$ such that $\Phi_{\mu \nu}\left(\mu^{\prime}, \nu^{\prime}\right)$ has bending measured lamination $\left(\mu^{\prime}, \nu^{\prime}\right)$ for every $\left(\mu^{\prime}, \nu^{\prime}\right) \in$ $R_{\mu \nu}$ and such that $\check{\mathcal{P}}^{+}(\mu)$ and $\check{\mathcal{P}}^{-}(\nu)$ meet transversely along $\beta\left(R_{\mu \nu}\right)$. Here, the statement that $R_{\mu \nu}$ is maximal means that $\left.\left.\varepsilon_{\mu \nu} \in\right] 0, \infty\right]$ is maximal for this property.

Note that $R_{\mu \nu}$ and $\varphi_{\mu \nu}$ depend only on the orbit of $(\mu, \nu)$ under the action of $\mathbb{R}^{+}$, namely on the corresponding point of $\partial \check{\mathcal{F}} \mathcal{M L}(S)$. Let $\check{R}_{\mu \nu}$ be the lift of $R_{\mu \nu}$ in $\check{\mathcal{F}} \mathcal{M L}(S)$, and lift $\Phi_{\mu \nu}$ to $\check{\Phi}_{\mu \nu}: \check{R}_{\mu \nu} \rightarrow \check{\mathcal{Q}}(S)$. In particular, $\check{\Phi}_{\mu \nu}$ sends the initial point of $\check{R}_{\mu \nu}$ to the bending vector $\bar{b}_{m}^{\mu}=\bar{b}_{m}^{-\nu} \in N^{1} \mathcal{F}(S)=\partial \check{\mathcal{Q}}(S)$ with $m=\kappa(\mu, \nu)$.

Let $\check{U} \subset \check{\mathcal{F}} \mathcal{M L}(S)$ denote the union of all the $\check{R}_{\mu \nu}$, and let $\check{\Phi}: \check{U} \rightarrow \mathcal{F} \mathcal{M L}(S)$ restrict to $\check{\Phi}_{\mu \nu}$ on each $\check{R}_{\mu \nu}$. Note that the property that $\Phi_{\mu \nu}\left(\mu^{\prime}, \nu^{\prime}\right)$ has bending measured lamination $\left(\mu^{\prime}, \nu^{\prime}\right)$ implies that the $\check{R}_{\mu \nu}$ are pairwise disjoint, so that $\check{\Phi}$ is well-defined. We want to show that $\check{\Phi}$ is continuous.

Lemma 12. As the measured geodesic lamination $\mu$ tends to $\mu_{0}$ for the topology of $\mathcal{M L}(S)$, the submanifold $\mathcal{P}^{ \pm}(\mu)$ tends to $\mathcal{P}^{ \pm}\left(\mu_{0}\right)$ for the topology of $\mathrm{C}^{\infty}$ convergence on compact subsets.

Proof. We will use the tools developed in Bo1.

Let $\mu_{n} \in \mathcal{M L}(S), n \in \mathbb{N}$, be a sequence converging to $\mu_{0}$. Let $\lambda_{n}$ be a maximal geodesic lamination containing the support of $\mu_{n}$. Passing to a subsequence if necessary, we can assume that, for the Hausdorff topology, the geodesic lamination $\lambda_{n}$ converges to a geodesic lamination $\lambda_{0}$, which is necessarily maximal and contains the support of $\mu_{0}$.

The shear-bend coordinates associated to $\lambda_{n}$ provide an open biholomorphic embedding $\Phi_{n}: \mathcal{Q}(S) \rightarrow \mathcal{H}_{0}\left(\lambda_{n} ; \mathbb{C} / 2 \pi \mathrm{i} \mathbb{Z}\right)$. Here $\mathcal{H}_{0}\left(\lambda_{n} ; \mathbb{C} / 2 \pi \mathrm{i} \mathbb{Z}\right)$ is the topological group of $\mathbb{C} / 2 \pi \mathrm{i} \mathbb{Z}$-valued transverse cocycles for the maximal geodesic lamination $\lambda_{n}$ which satisfy the cusp condition, and is isomorphic to $(\mathbb{C} / 2 \pi \mathrm{i} \mathbb{Z})^{\theta} \oplus \mathbb{Z} / 2$. For a metric $m \in \mathcal{Q}(S)$, the real part of $\Phi_{n}(m) \in \mathcal{H}_{0}\left(\lambda_{n} ; \mathbb{C} / 2 \pi \mathrm{i} \mathbb{Z}\right)=\mathcal{H}_{0}\left(\lambda_{n} ; \mathbb{R}\right) \oplus$ $i \mathcal{H}_{0}\left(\lambda_{n} ; \mathbb{R} / 2 \pi \mathbb{Z}\right)$ measures the induced metric of the unique $m$-pleated surface with bending locus $\lambda_{n}$, and the imaginary part measures its bending. In particular, $\mathcal{P}^{ \pm}\left(\mu_{n}\right)$ locally corresponds to $\Phi_{n}^{-1}\left(\mathcal{H}_{0}\left(\lambda_{n} ; \mathbb{R}\right) \oplus \mathrm{i} \mathbb{R} \mu_{n}\right)$, or more precisely to a branch of the immersion which is the composition of the projection $\mathcal{H}_{0}\left(\lambda_{n} ; \mathbb{R}\right) \oplus \mathrm{i} \mathbb{R} \mu_{n} \rightarrow \mathcal{H}_{0}\left(\lambda_{n} ; \mathbb{C} / 2 \pi \mathrm{i} \mathbb{Z}\right)$ and of $\Phi_{n}^{-1}$.

To compare the various $\mathcal{H}_{0}\left(\lambda_{n} ; \mathbb{C} / 2 \pi \mathrm{i} \mathbb{Z}\right)$ pick a train track $\tau$ carrying $\lambda_{0}$. Since $\lambda_{n}$ converges to $\lambda_{0}$ for the Hausdorff topology, $\tau$ also carries the $\lambda_{n}$ for $n$ large enough. Then there is a well-defined isomorphism $\Psi_{n}: \mathcal{H}_{0}\left(\lambda_{n} ; \mathbb{C} / 2 \pi \mathrm{i} \mathbb{Z}\right) \rightarrow$ 
$\mathcal{W}(\tau ; \mathbb{C} / 2 \pi \mathrm{i} \mathbb{Z})$, where $\mathcal{W}(\tau ; \mathbb{C} / 2 \pi \mathrm{i} \mathbb{Z})$ is the group of $\mathbb{C} / 2 \pi \mathrm{i} \mathbb{Z}$-valued edge weights for $\tau$ satisfying the switch and cusp relations.

Because $\lambda_{n}$ converges to $\lambda_{0}$ for the Hausdorff topology, if follows from the explicit construction of [Bo1, $\S 5, \S 8]$ that $\Phi_{n}^{-1} \circ \Psi_{n}^{-1}$ converges to $\Phi_{0}^{-1} \circ \Psi_{0}^{-1}$, uniformly on compact subsets of the image of $\Psi_{0} \circ \Phi_{0}$. Because these maps are holomorphic, the convergence is actually $\mathrm{C}^{\infty}$. Since $\mu_{n}$ converges to $\mu_{0}$ for the topology of $\mathcal{M L}(S)$, the edge weight system $\Psi_{n}\left(\mu_{n}\right)$ converges to $\Psi_{0}\left(\mu_{0}\right)$ in $\mathcal{W}_{0}(\tau, \mathbb{R})$. It follows that $\mathcal{P}^{ \pm}\left(\mu_{n}\right)$, which locally corresponds to $\Phi_{n}^{-1} \circ \Psi_{n}^{-1}\left(\mathcal{W}_{0}(\tau ; \mathbb{R}) \oplus \mathrm{i} \mathbb{R} \Psi_{n}\left(\mu_{n}\right)\right)$, converges to $\mathcal{P}^{ \pm}\left(\mu_{0}\right)$, which locally corresponds to $\Phi_{0}^{-1} \circ \Psi_{0}^{-1}\left(\mathcal{W}_{0}(\tau ; \mathbb{R}) \oplus \mathrm{i} \mathbb{R} \Psi_{0}\left(\mu_{0}\right)\right)$, in the topology of $\mathrm{C}^{\infty}$-convergence on compact subsets.

Note that we actually proved real analytic convergence in Lemma 12. However, we will only need $\mathrm{C}^{2}$ convergence.

Theorem 13. The subset $\check{U}$ is an open neighborhood of $\partial \check{\mathcal{F}} \mathcal{M L}(S)$ in $\check{\mathcal{F}} \mathcal{M L}(S)$, and $\check{\Phi}$ is a homeomorphism from $\check{U}$ to an open neighborhood $\check{V}$ of $\partial \check{\mathcal{Q}}(S)$ in $\check{\mathcal{Q}}(S)$.

Proof. The restriction $\check{\Phi}_{\mu \nu}$ of $\check{\Phi}$ to $\check{R}_{\mu \nu}$ was constructed by considering the transverse intersection of the submanifolds $\check{\mathcal{P}}^{+}(\mu)$ and $\check{\mathcal{P}}^{-}(\nu)$ near the boundary of $\check{\mathcal{Q}}(S)$. By Lemma 12, $\check{\mathcal{P}}^{+}(\mu)$ and $\check{\mathcal{P}}^{-}(\nu)$ depend continuously on $(\mu, \nu)$ for the topology of $\mathrm{C}^{1}$ convergence. (Note that one needs the $\mathrm{C}^{2}$ continuity of $\mathcal{P}^{+}(\mu)$ and $\mathcal{P}^{-}(\nu)$ to guarantee the $\mathrm{C}^{1}$ continuity of $\check{\mathcal{P}}^{+}(\mu)$ and $\check{\mathcal{P}}^{-}(\nu)$ near the boundary.) It follows that the length $\varepsilon_{\mu \nu}$ of $R_{\mu \nu}=\left[0, \varepsilon_{\mu \nu}[(\mu, \nu)\right.$ is a lower semi-continuous function of $(\mu, \nu)$, and that $\Phi_{\mu \nu}$ depends continuously on $(\mu, \nu)$. This proves that the union $\check{U}$ of the $\check{R}_{\mu \nu}$ is open in $\partial \check{\mathcal{F}} \mathcal{M L}(S)$, and that $\check{\Phi}$ is continuous.

Let $p: \check{\mathcal{Q}}(S) \rightarrow \mathcal{Q}(S)$ be the natural projection and, as usual, let $\beta: \mathcal{Q}(S) \rightarrow$ $\mathcal{M L}(S)^{2}$ be the bending map. By construction, $\beta \circ p \circ \check{\Phi}$ is the identity map on $\check{U}-\partial \check{\mathcal{F}} \mathcal{M L}(S) \subset \mathcal{M L}(S)^{2}$. It follows that $\check{\Phi}$ is injective on $\check{U}-\partial \check{\mathcal{F}} \mathcal{M L}(S)$, and therefore on all of $\check{U}$ by Proposition 6 .

The two spaces $\check{U} \subset \check{\mathcal{F}} \mathcal{M L}(S)$ and $\check{\mathcal{Q}}(S)$ are topological manifolds-withboundary of the same dimension $2 \theta$. The map $\check{\Phi}: \check{U} \rightarrow \mathcal{F} \mathcal{M L}(S)$ is continuous and injective, and sends boundary points to boundary points. By the Theorem of Invariance of the Domain, it follows that its image $\check{V}=\check{\Phi}(\check{U})$ is open in $\check{\mathcal{Q}}(S)$, and that $\check{\Phi}$ restricts to a homeomorphism $\check{U} \rightarrow \check{V}$.

Theorem 14. There exists an open neighborhood $V$ of the fuchsian submanifold $\mathcal{F}(S)$ in $\mathcal{Q}(S)$ such that the bending map $\beta: \mathcal{Q}(S) \rightarrow \mathcal{M L}(S)^{2}$ induces a homeomorphism between $V-\mathcal{F}(S)$ and its image.

Proof. For the canonical identifications between $\check{\mathcal{Q}}(S)-\partial \check{\mathcal{Q}}(S)$ and $\mathcal{Q}(S)-\mathcal{F}(S)$, and between $\check{\mathcal{F}} \mathcal{M L}(S)-\partial \check{\mathcal{F}} \mathcal{M L}(S)$ and $\mathcal{F} \mathcal{M L}(S)$, the inverse of the restriction of $\check{\Phi}$ to $\check{U}-\partial \check{\mathcal{Q}}(S)$ coincides with $\beta$. Therefore, the only part which requires some checking is that the image $V$ of $\check{V}=\check{\Phi}(\check{U})$ under the canonical projection $p: \check{\mathcal{Q}}(S) \rightarrow \mathcal{Q}(S)$ is open in $\mathcal{Q}(S)$. However, this immediately follows from the fact that the preimages of points under $p$ are all compact, which implies that $p$ is an open map. (Note that this is false for the projection $\check{\mathcal{F}} \mathcal{M L}(S) \rightarrow \mathcal{F} \mathcal{M L}(S) \cup\{0\}$.)

Theorem 14 is just Theorem 1 stated in the introduction. Theorem 2 immediately follows from the definition of $U$ and from the fact that $\check{U}$ is open (Theorem 13).

We conclude with a few remarks. 
A recent result of Caroline Series Se2] shows that we can restrict the neighborhood $V$ of Theorem 14 so that $\beta^{-1}(\beta(V))=V$.

When $\mu$ and $\nu$ are multicurves, namely when their supports consist of finitely many closed geodesics, it follows from [HoK] that the submanifolds $\check{\mathcal{P}}^{+}(\mu)$ and $\check{\mathcal{P}}^{-}(\nu)$ are everywhere transverse, by a doubling argument as in BoO. Consequently, the open subset $V$ of Theorem 14 can be chosen so that the image $U=\beta(V) \subset \mathcal{M L}(S)^{2}$ contains all rays of the form $[0, \infty[(\mu, \nu)$ where $\mu$ and $\nu$ are multicurves.

As indicated in the introduction, it is conjectured that we can take $V$ equal to the whole space $\mathcal{Q}(S)$. See $\mathrm{BoO}$ for a characterization of the image of $\mathcal{Q}(S)$ under $\beta$.

\section{REFERENCES}

[Bo1] Francis Bonahon, Shearing hyperbolic surfaces, bending pleated surfaces and Thurston's symplectic form, Ann. Fac. Sci. Toulouse Math. (6) 5 (1996), pp. 233-297.

[Bo2] Geodesic laminations with transverse Hölder distributions, Ann. Sci. École Norm. Sup. (4) 30 (1997), pp. 205-240.

[Bo3] - The boundary geometry of 3-dimensional hyperbolic convex cores, J. Differential Geometry 50 (1998), pp. 1-23.

[BoO] Francis Bonahon, Jean-Pierre Otal, Laminations mesurées de plissage des variétés hyperboliques de dimension 3, preprint, 2001.

[EpM] David B. A. Epstein, Albert Marden, Convex hulls in hyperbolic spaces, a theorem of Sullivan, and measured pleated surfaces, in: Analytical and geometric aspects of hyperbolic space (D.B.A. Epstein ed.), L.M.S. Lecture Note Series vol. 111, 1986, Cambridge University Press, pp. 113-253.

[HoK] Craig D. Hodgson, Steven P. Kerckhoff, Rigidity of hyperbolic cone-manifolds and hyperbolic Dehn surgery, J. Differential Geom. 48 (1998), pp. 1-59.

[KaT] Yoshinobu Kamishima, Ser P. Tan, Deformations spaces of geometric structures, in: Aspects of low-dimensional manifolds (Y. Matsumo, S. Morita eds.), Advanced Studies in Pure Math. 20, 1992, Kinokuniya Company Ltd., Tokyo, pp. 263-299.

[Ke1] Steven P. Kerckhoff, The Nielsen realization problem, Ann. of Math. 117 (1983), pp. 235265.

[Ke2] L Lines of minima in Teichmüller space, Duke Math. J. 65 (1992), pp. 187-213.

[Mar] Albert Marden, The geometry of finitely generated Kleinian groups, Ann. of Math. 99 (1974), pp. 383-462.

[Mas] Bernard Maskit, Kleinian groups, Grundlehren der Math. Wiss. 287, Springer-Verlag, 1988.

[Se1] Caroline M. Series, On Kerckhoff minima and pleating loci for quasi-Fuchsian groups, Geom. Dedicata 88 (2001), pp. 211-237.

[Se2] L Limits of quasifuchsian groups with small bending, preprint, 2002.

[Su] Dennis P. Sullivan, Quasiconformal homeomorphisms and dynamics II: Structural stability implies hyperbolicity for Kleinian groups, Acta Math. 155 (1985), pp. 243-260.

[Th1] William P. Thurston, The topology and geometry of 3-manifolds, Lecture notes, Princeton University, 1976-79.

[Th2] — Earthquakes in two-dimensional hyperbolic geometry, in: Low-dimensional Topology and Kleinian groups (D.B.A. Epstein ed.), L.M.S. Lecture Notes Series vol. 112, 1986, Cambridge University press, pp. 91-112.

[Wo1] Scott A. Wolpert, On the symplectic geometry of deformations of a hyperbolic surface, Ann. of Math. 117 (1983), pp. 207-234.

[Wo2] $\longrightarrow$ Geodesic length functions and the Nielsen problem, J. Differential Geom. 25 (1987), pp. 275-296. 
F. Bonahon, Department of Mathematics, University of Southern California, Los ANGeles, CA 90089-1113, U.S.A.

E-mail address: fbonahon@math.usc.edu

$U R L:$ http://math.usc.edu/ ${ }^{\text {fbonahon }}$ 